\title{
Hospital-wide surveillance-based antimicrobial treatments: a Monte-Carlo look-ahead method
}

\author{
Nicolas HouY* Julien Flaig ${ }^{\dagger}$
}

23rd January 2021

\begin{abstract}
Background and objectives. We present a heuristic solution method to the problem of choosing hospital-wide antimicrobial treatments that minimize the cumulative infected patient-days in the long run in a health care facility.

Methods. Our solution method is a rollout algorithm. We rely on the stochastic version of a compartmental model to describe the spread of an infecting organism in the health care facility and the emergence and spread of resistance to two drugs. We assume that the parameters of the model are known. Treatments are chosen at the beginning of each period based on the count of patients with each health status, and on stochastic simulations of the future emergence and spread of antimicrobial resistance. The same treatment is then administered to all patients, including uninfected patients, during the period and cannot be adjusted until the next period.

Results. In our simulations, our algorithm allows to reduce the average cumulative infected patient-days over two years by $47.0 \%$ compared to the best standard therapy, and by $32.2 \%$ compared to a similar heuristic algorithm not using surveillance data (significantly at the $95 \%$ threshold).

Conclusion. Our heuristic solution method is simple yet flexible. We explain how it can be used either to perform online optimization, or to produce data for quantitative analysis. Its performance is illustrated using a relatively simple infectious disease transmission model, but it is compatible with more advanced epidemiological models.
\end{abstract}

Keywords: health care associated infection, antimicrobial resistance, antibiotic cycling, optimization, Monte-Carlo methods, stochastic model.

\footnotetext{
*University of Lyon, Lyon, F-69007, France; CNRS, GATE Lyon Saint-Etienne, F-69130, France. Email: houy@gate.cnrs.fr.

${ }^{\dagger}$ Email: julien@jflaig.com. No financial support was provided for this study.
} 


\section{Introduction}

Health care facilities are particularly affected by infections with drug resistant pathogens, a major cause of morbidity and mortality [8]. There are two main reasons for this. First, the spread of infections (whether drug resistant or not) can be easier in hospital environments [14] and furthermore hospital patients are more at risk of serious complications than the general population. Second, antimicrobials are heavily used in health care facilities, both for preventive reasons and empirically. Empirical therapies are antimicrobial treatments administered at the onset of symptoms, when a therapy needs to be started before microbiological test results are available. In this situation, the causative pathogen and its resistance status can be entirely or partly unknown. However under antimicrobial treatment, drug resistant mutant strains are favored compared to drug susceptible strains. Antimicrobials can select for resistance both at the within-host and between-host levels [17], thus prompting the emergence of resistance in health care facilities or the spread of imported resistant strains.

In order to tackle this issue, empirical prescription policies have been proposed to slow and even revert the evolution of resistance [19]. Indeed, at the molecular level, resistance is acquired through the modification of essential pathways of the pathogenic organism. Therefore, it is thought to be often associated with fitness costs: in the absence of treatment, resistant organisms are less able to survive and reproduce than wild-type susceptible organisms $[2,18]$. It was then hoped that empirical therapy policies such as drug mixing - assigning drugs randomly to patients - or drug cycling - switching from one drug to another following a rotation schedule - would limit the emergence and spread of antimicrobial resistance. Yet the respective merits of drug mixing and drug cycling have been debated, and empirical studies remain inconclusive (see [3, 4, 7, 16, 21, 22, 24] and the references therein). The theoretical investigations of empirical therapy policies and their

design typically rely on aggregate (between-host) models of the emergence and spread of antimicrobial resistance. We refer to $[6,20,23]$ for extensive reviews of these models and their applications.

Strategies making use of available information to adapt treatments (which is not the case of mixing and cycling) have been proposed. In particular, it was shown that hospitalwide policies based on the results of microbiological tests could limit the spread of resistance [15] (see [1] for an example of similar strategy used in the field). In this scenario, all symptomatic patients are tested at a given rate in order to choose an adequate individual treatment. Asymptomatic patients and not yet tested symptomatic patients receive an empirical treatment based on available test results. A significant advantage of this strategy is 
that it makes use of information available at no additional cost since tests are performed by default for all symptomatic patients. Notice that hospital-wide policies are to be contrasted from adaptation of treatments at the individual level. It was show than adapting individual treatments based on the apparition of symptoms can outperform mixing and cycling [27]; and that using individual test results to adapt individual treatments can outperform not only mixing and cycling, but also hospital-wide treatments based on test results at the population level [3]. However, strategies at the individual level are left outside the scope of this article. We focus on strategies at the hospital level. Such strategies have received little attention outside [3, 15].

In the present article, we want to look at the problem of devising hospital-wide treatment policies based on microbiological test results from a different angle and to propose a new method to solve this problem. Our solution method has two important features. First, we strive for near optimal policies. By contrast, the policies presented in [15], for instance, are adaptive in that they specify which empirical treatment is to be administered based solely on test results available at that time. Test results only depend on the spread of the disease in the past and on past treatments, and decisions do not anticipate future scenarios. Of course, the merit of an empirical therapy at a given time depends not only on past treatments, but also on the subsequent course of the epidemic, that is on subsequent empirical treatments. We solve this dynamic optimization problem using a rollout algorithm [5, 9]. This heuristic algorithm consists in choosing an empirical treatment for a given period based on simulations of the future emergence and spread of resistant pathogens in the considered health care facility. To do this we rely on a stochastic compartmental model derived from models previously introduced in [23]. Our hospital-wide treatment policies are contingent on the exact number of patients with each health status, so relying on a stochastic model is necessary. Simulations assume future treatments heuristically. At this point, it is worth emphasizing that our objective is not to propose a new epidemiological model or to investigate the forces driving the emergence and spread of antimicrobial resistance in health care facilities. We use a theoretical compartmental model meant to reproduce general features of the emergence and spread of antimicrobial resistance, rather than the features of a particular pathogen or health care facility. The Monte-Carlo look-ahead procedure presented in this article was previously used to design empirical antimicrobial therapy policies based on the same compartmental model in scenarios where surveillance data is not available [11]. Similar algorithms were also used to optimize chemotherapy regimens $[12,13]$.

Second, we assume that we periodically have access to the health status of all patients in the hospital. That is, we are able to test all patients with a fixed period, and test results 
are available immediately. ${ }^{1}$ In [15], information becomes available continuously, but it is only partial - test results are used as a proxy for actual prevalence. Also, the hospitalwide treatment policies we propose to devise go beyond the strict definition of empirical treatments, although our study stems from the empirical treatment literature. Indeed, we assume that all patients, including uninfected patients, receive the same treatment; and that treatments are not continuously adjusted dynamically during periods between observations. In particular, patients whose health status is known receive all the same treatment. This raises obvious ethical issues that will not be discussed here: the flexibility of our heuristic method allows to easily take into account any ethical or practical constraint.

We present the materials and methods used in the study in Section 2: the compartmental model describing the emergence and spread of a microbial disease with resistant strains in a health care facility in Section 2.1, and the optimization algorithm in Section 2.2. We show and discus our results in Section 3. Section 4 concludes.

\section{Materials and methods}

\subsection{Model}

A sketch of our epidemiological model is shown in Figure 1. We refer to [11] for an introduction of the model and an in-depth discussion of the underlying biological assumptions. We summarize the considered events in Table 1 and the parameters in Table 2. Notice that we do not seek to model the spread of a specific pathogen, which is standard practice in the theoretical and methodological literature [23]. Parameter values were chosen in biologically plausible ranges; see for instance [26]. We emphasize that methods for learning parameters values from data are left outside the scope of this study. The model was used in a previous study [10] to underline the difference between decision making when parameters are unknown, and decision making when they are known as it is the case in the present study.

A summary of the main features of the model is adapted from [10] below.

- Pathogenic strains. We model resistance to two drugs, drug 1 and drug 2. At each time, a patient is either infected with a wild-type strain susceptible to both drugs, infected with a strain resistant to drug 1 but not to drug 2 (1-resistant), infected with a strain resistant to drug 2 but not to drug 1 (2-resistant), infected with a strain

\footnotetext{
${ }^{1}$ The issue of data acquisition and integration is not considered here. We refer to [25] for a broad overview of the use of artificial intelligence in the design of drug treatments.
} 
resistant to both drugs (12-resistant), or uninfected with any of the above. $S, R_{1}$, $R_{2}, R_{12}$, and $X$ denote the number of patients with each health status respectively. We assume that infected patients are only infected with one pathogenic strain at any given time.

- Admissions and discharges. We consider a small health care facility or a hospital ward with an average population of $N=40$ patients. We assume that admission and discharge do not depend on health status. However, we also make the simplifying assumption that transmission of strains and emergence of resistance in the community is exogenous to the problem in hand.

- Treatments. Three treatments are available: drug 1 in monotherapy (treatment 1), drug 2 in monotherapy (treatment 2), and a combination of drugs 1 and 2 (treatment 12). Patients can also be left without a treatment (treatment 0 ). Decision variable $f_{i}, i \in\{0,1,2,12\}$, is equal to 1 when patients receive treatment $i$ and to 0 otherwise. At all time, $f_{0}+f_{1}+f_{2}+f_{12}=1$.

- Recovery. Infected patients recover spontaneously at rate $\gamma$ regardless of the infecting strain. Patients receiving adequate treatment recover at rate $\tau$.

- De novo mutation. Infecting organisms acquire $i$-resistance through de novo mutation at rate $\nu_{i}, i \in\{1,2,12\}$, of two different genes as illustrated in Figure A.1 in Appendix.

- Infection and superinfection. We assume homogeneous mixing. Pathogenic strains are transmitted to uninfected patients with transmission rate $\beta$, and to already infected patients with transmission rate $\sigma \beta$. We assume $\sigma=1$ in this instance of the model, but the general case is $\sigma \in[0,1]$. $i$-resistant strains incur a fitness cost on transmission $c_{i} \in[0,1], i \in\{1,2,12\}$, and their transmission rate is weighed by $1-c_{i}$.

- Strain replacement. Within-host, a new pathogenic strain acquired through de novo mutation or contagion must compete with the resident pathogenic strain or with the commensal microflora in the case of previously uninfected patients. $i$-resistant strains incur a fitness cost $s_{i} \in[0,1], i \in\{1,2,12\}$, on competitive performance. We normalize the fitness cost $s_{S}$ of the wild-type susceptible strain to 0 , and the fitness cost $s_{X}$ of the commensal microflora to 1 . A strain receiving adequate treatment has a fitness cost of 1 . The rate of replacement of a resident strain with fitness cost $s_{j}$ 


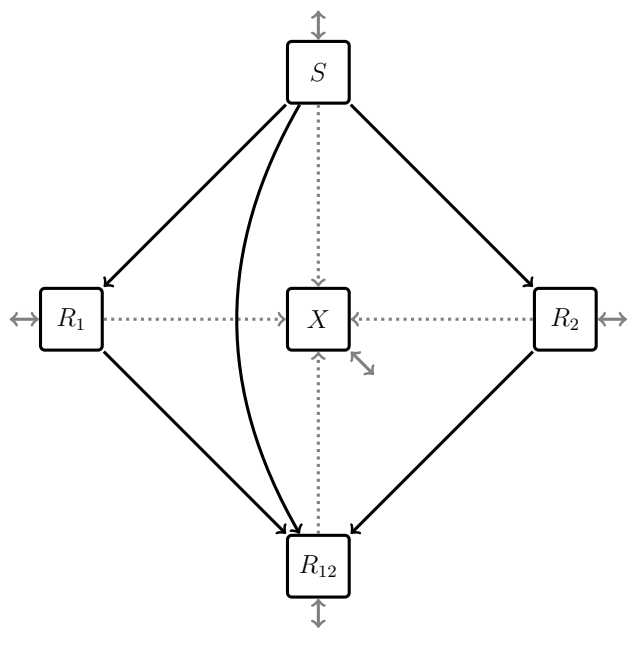

(a) De novo mutations.

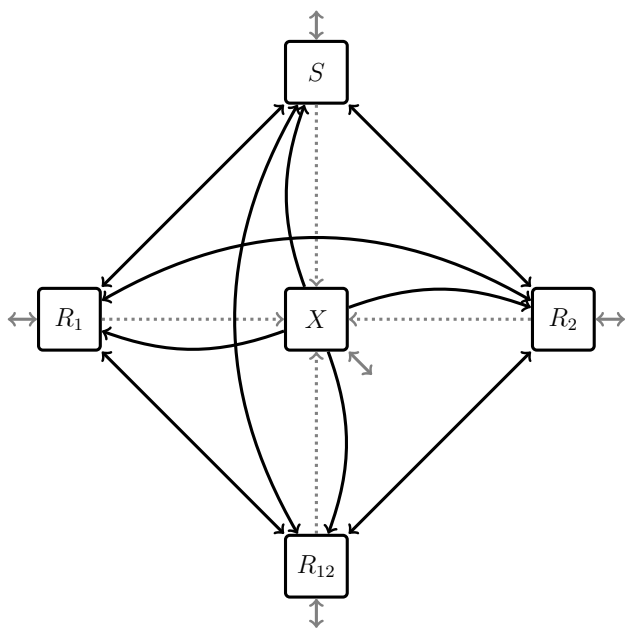

(b) Contagion.

Figure 1: Compartmental model. For clarity we display de novo mutations (black, panel (a)) and contagion (black, panel (b)) on two separate graphs. Gray: admissions and discharges. Dotted gray: recovery. Some arrows representing different events were merged for readability. Reproduced from [10].

by a new strain with fitness cost $s_{i}$ is given by function $\chi_{\epsilon}$ as

$$
\chi_{\epsilon}\left(s_{i}, s_{j}\right)= \begin{cases}1 & \text { if } s_{i}-s_{j} \leq-\epsilon \\ 0.5-\frac{s_{i}-s_{j}}{2 \epsilon} & \text { if }\left|s_{i}-s_{j}\right|<\epsilon \\ 0 & \text { if } s_{i}-s_{j} \geq \epsilon .\end{cases}
$$

We further assume that a new strain cannot colonize a treated patient unless it is resistant to the treatment. 


\begin{tabular}{|c|c|}
\hline Population changes & Rate \\
\hline \multicolumn{2}{|l|}{ Admissions and discharges } \\
\hline$S \leftarrow S+1$ & $N \mu m_{S}$ \\
\hline$R_{i} \leftarrow R_{i}+1, i \in\{1,2,12\}$ & $N \mu m_{i}$ \\
\hline$X \leftarrow X+1$ & $N \mu\left(1-\sum_{i \in\{S, 1,2,12\}} m_{i}\right)$ \\
\hline$S \leftarrow S-1$ & $\mu S$ \\
\hline$R_{i} \leftarrow R_{i}-1, i \in\{1,2,12\}$ & $\mu R_{i}$ \\
\hline$X \leftarrow X-1$ & $\mu X$ \\
\hline \multicolumn{2}{|l|}{ De novo mutations } \\
\hline$S \leftarrow S-1, R_{i} \leftarrow R_{i}+1, i \in\{1,2\}$ & $\nu_{i}\left[f_{0} \chi_{\epsilon}\left(s_{i}, s_{S}\right)+f_{i} \chi_{\epsilon}\left(s_{i}, 1\right)\right] S$ \\
\hline$S \leftarrow S-1, R_{12} \leftarrow R_{12}+1$ & $\nu_{12}\left[f_{0} \chi_{\epsilon}\left(s_{12}, s_{S}\right)+\left(f_{1}+f_{2}+f_{12}\right) \chi_{\epsilon}\left(s_{12}, 1\right)\right] S$ \\
\hline$R_{i} \leftarrow R_{i}-1, R_{12} \leftarrow R_{12}+1, i \in\{1,2\}$ & $\begin{aligned} \nu_{(3-i)}\left[\left(f_{0}+f_{i}\right) \chi_{\epsilon}\left(s_{12}, s_{i}\right)\right. & \\
& \left.+\left(f_{(3-i)}+f_{12}\right) \chi_{\epsilon}\left(s_{12}, 1\right)\right] R_{i}\end{aligned}$ \\
\hline \multicolumn{2}{|l|}{ Infections and recoveries } \\
\hline$X \leftarrow X-1, S \leftarrow S+1$ & $f_{0} \beta\left(1-c_{S}\right) \chi_{\epsilon}\left(s_{S}, 1\right) X S / N$ \\
\hline$X \leftarrow X-1, R_{i} \leftarrow R_{i}+1, i \in\{1,2\}$ & $\left(f_{0}+f_{(3-i)}\right) \beta\left(1-c_{i}\right) \chi_{\epsilon}\left(s_{i}, 1\right) X R_{i} / N$ \\
\hline$X \leftarrow X-1, R_{12} \leftarrow R_{12}+1$ & $\beta\left(1-c_{12}\right) \chi_{\epsilon}\left(s_{12}, 1\right) X R_{12} / N$ \\
\hline$S \leftarrow S-1, X \leftarrow X+1$ & {$\left[\tau\left(f_{1}+f_{2}+f_{12}\right)+\gamma\right] S$} \\
\hline$R_{i} \leftarrow R_{i}-1, X \leftarrow X+1, i \in\{1,2\}$ & {$\left[\tau\left(f_{(3-i)}+f_{12}\right)+\gamma\right] R_{i}$} \\
\hline$R_{12} \leftarrow R_{12}-1, X \leftarrow X+1$ & $\gamma R_{12}$ \\
\hline \multicolumn{2}{|l|}{ Superinfections } \\
\hline$S \leftarrow S-1, R_{i} \leftarrow R_{i}+1, i \in\{1,2\}$ & $\sigma \beta\left(1-c_{i}\right)\left[f_{0} \chi_{\epsilon}\left(s_{i}, s_{S}\right)+f_{i} \chi_{\epsilon}\left(s_{i}, 1\right)\right] S R_{i} / N$ \\
\hline$R_{i} \leftarrow R_{i}-1, S \leftarrow S+1, i \in\{1,2\}$ & $\sigma \beta\left(1-c_{S}\right) f_{0} \chi_{\epsilon}\left(s_{S}, s_{i}\right) S R_{i} / N$ \\
\hline$S \leftarrow S-1, R_{12} \leftarrow R_{12}+1$ & $\begin{array}{l}\sigma \beta\left(1-c_{12}\right)\left[f_{0} \chi_{\epsilon}\left(s_{12}, s_{S}\right)\right. \\
\left.\quad+\left(f_{1}+f_{2}+f_{12}\right) \chi_{\epsilon}\left(s_{12}, 1\right)\right] S R_{12} / N\end{array}$ \\
\hline$R_{12} \leftarrow R_{12}-1, S \leftarrow S+1$ & $\sigma \beta\left(1-c_{S}\right) f_{0} \chi_{\epsilon}\left(s_{S}, s_{12}\right) S R_{12} / N$ \\
\hline$R_{i} \leftarrow R_{i}-1, R_{12} \leftarrow R_{12}+1, i \in\{1,2\}$ & $\begin{aligned} \sigma \beta\left(1-c_{12}\right) & {\left[\left(f_{0}+f_{i}\right) \chi_{\epsilon}\left(s_{12}, s_{i}\right)\right.} \\
& \left.+\left(f_{(3-i)}+f_{12}\right) \chi_{\epsilon}\left(s_{12}, 1\right)\right] R_{i} R_{12} / N\end{aligned}$ \\
\hline$R_{12} \leftarrow R_{12}-1, R_{i} \leftarrow R_{i}+1, i \in\{1,2\}$ & $\sigma \beta\left(1-c_{i}\right)\left(f_{0}+f_{i}\right) \chi_{\epsilon}\left(s_{i}, s_{12}\right) R_{i} R_{12} / N$ \\
\hline $\begin{array}{l}R_{i} \leftarrow R_{i}-1, \\
\quad R_{(3-i)} \leftarrow R_{(3-i)}+1, i \in\{1,2\}\end{array}$ & $\begin{aligned} \sigma \beta\left(1-c_{(3-i)}\right) & {\left[f_{0} \chi_{\epsilon}\left(s_{(3-i)}, s_{i}\right)\right.} \\
& \left.+f_{(3-i)} \chi_{\epsilon}\left(s_{(3-i)}, 1\right)\right] R_{i} R_{(3-i)} / N\end{aligned}$ \\
\hline
\end{tabular}

Table 1: Events considered in the model. 


\begin{tabular}{|c|c|c|c|}
\hline Parameter & Unit & Description & Value \\
\hline \multicolumn{4}{|c|}{ Admissions and discharges } \\
\hline$N$ & - & Average number of patients. & 40 \\
\hline$\mu$ & $\operatorname{day}^{-1}$ & Turnover rate. & 0.1 \\
\hline$m_{S}$ & - & $\begin{array}{l}\text { Proportion of incoming patients infected with the sus- } \\
\text { ceptible strain. }\end{array}$ & 0.1 \\
\hline$m_{1}$ & - & $\begin{array}{l}\text { Proportion of incoming patients infected with the } \\
\text { strain resistant to drug } 1 .\end{array}$ & 0.05 \\
\hline$m_{2}$ & - & $\begin{array}{l}\text { Proportion of incoming patients infected with the } \\
\text { strain resistant to drug } 2 \text {. }\end{array}$ & 0.01 \\
\hline$m_{12}$ & - & $\begin{array}{l}\text { Proportion of incoming patients infected with the } \\
\text { strain resistant to drugs } 1 \text { and } 2 .\end{array}$ & 0.005 \\
\hline \multicolumn{4}{|c|}{ De novo mutations and fitness costs } \\
\hline$\nu_{i}$ & day $^{-1}$ & $\begin{array}{l}\text { Rate of acquisition of resistance to treatment } i \text { through } \\
\text { de novo mutation. } i \in\{1,2,12\}\end{array}$ & 0.005 \\
\hline$c_{i}$ & - & $\begin{array}{l}\text { Cost on infectivity of resistance to treatment } i, i \in \\
\{1,2,12\} \text {. }\end{array}$ & 0.2 \\
\hline$c_{S}$ & - & Cost on infectivity of susceptible organisms. & 0 \\
\hline$s_{i}$ & - & $\begin{array}{l}\text { Cost on competitive performance of resistance to treat- } \\
\text { ment } i, i \in\{1,2,12\} \text {. }\end{array}$ & 0.2 \\
\hline$s_{S}$ & - & $\begin{array}{l}\text { Cost on competitive performance of susceptible organ- } \\
\text { isms. }\end{array}$ & 0 \\
\hline$s_{X}$ & - & $\begin{array}{l}\text { Cost on competitive performance of commensal organ- } \\
\text { isms. }\end{array}$ & 1 \\
\hline$\epsilon$ & - & Slope parameter in function $\chi_{\epsilon}$. & 0.5 \\
\hline \multicolumn{4}{|c|}{ Infections, superinfections, and recoveries } \\
\hline$\beta$ & day $^{-1}$ & Transmission rate. & 0.5 \\
\hline$\sigma$ & - & Relative rate of superinfection. & 1 \\
\hline$\gamma$ & day $^{-1}$ & Rate of spontaneous recovery. & 0.1 \\
\hline$\tau$ & day $^{-1}$ & Rate of recovery under appropriate treatment. & 0.3 \\
\hline
\end{tabular}

Table 2: Model parameters. 


\subsection{Optimization algorithm}

Our objective is to minimize the cumulative infected patient-days in the long run (two years). We assume that we are able to know the exact number of patients with each health status every $\Delta h$ days. Upon observation of patients' health status, we must decide on a treatment to be administered to all patients over the next $\Delta h$ days. This treatment cannot be changed over $\Delta h$ days. This precludes adjustments of the treatment administered to all patients, but also adjustments of individual treatments based, for instance, on the evolution of the symptoms. In the following, unless otherwise specified, $\Delta h=15$ days.

Function StateToTREatment (Algorithm 1) returns the treatment to be used over the next $\Delta h$ days given the observed current state of the epidemic. ${ }^{2} \Theta$ denotes the set of available treatments among which we are to choose. It includes treatments $0,1,2$, and 12. The treatment administered during the next $\Delta h$ days will have an influence on the subsequent spread of the disease, on the emergence of drug resistance, and therefore on the cumulative infected patient-days. However, the long term effects of this treatment will also depend on subsequent treatment decisions, which are yet unknown. Therefore, we decide on the treatment to be administered during the next $\Delta h$ days by heuristically assuming that a default treatment $\theta_{\text {default }}$ will be administered afterwards over an horizon $H-\Delta h$, where $H$ is equal to two years (720 days).

Since the population dynamics parameters of the health care facility and of the disease are known, we are able to compare treatments by running stochastic simulations of the model presented in Section 2.1, starting from the observed state of the epidemic. This is done in the two basic functions stochSimu and EvaluateDecision that consist in computing a score over a set of stochastic simulations. ${ }^{3}$

For each available treatment option $\theta \in \Theta$, function stateToTREATMEnT runs $n_{S}=$ 800 stochastic simulations assuming that $\theta$ is administered over $\Delta h$ days, and that default treatment $\theta_{\text {default }}$ is administered during the following $H-\Delta h$ days. We choose the treatment $\theta^{*}$ for which the average over $n_{S}$ simulations of the cumulative infected patientdays over $H$ is the smallest.

The results presented in this article were obtained using treatment 2 as default treatment. Notice that different default treatments will lead to different decisions. A default treatment is chosen beforehand by simulating the use of the algorithm with different default treatments. Which default treatment performs better than the others will depend on

\footnotetext{
${ }^{2}$ Our dynamic model is Markovian, so all the information regarding the past dynamics is indeed summarized with no loss in the current state of the epidemic.

${ }^{3}$ Both functions are implemented identically when solving the problem without observing the state of the epidemic (see [11]), but in the present case their output is used online.
} 
the underlying epidemiological model and its parameters.

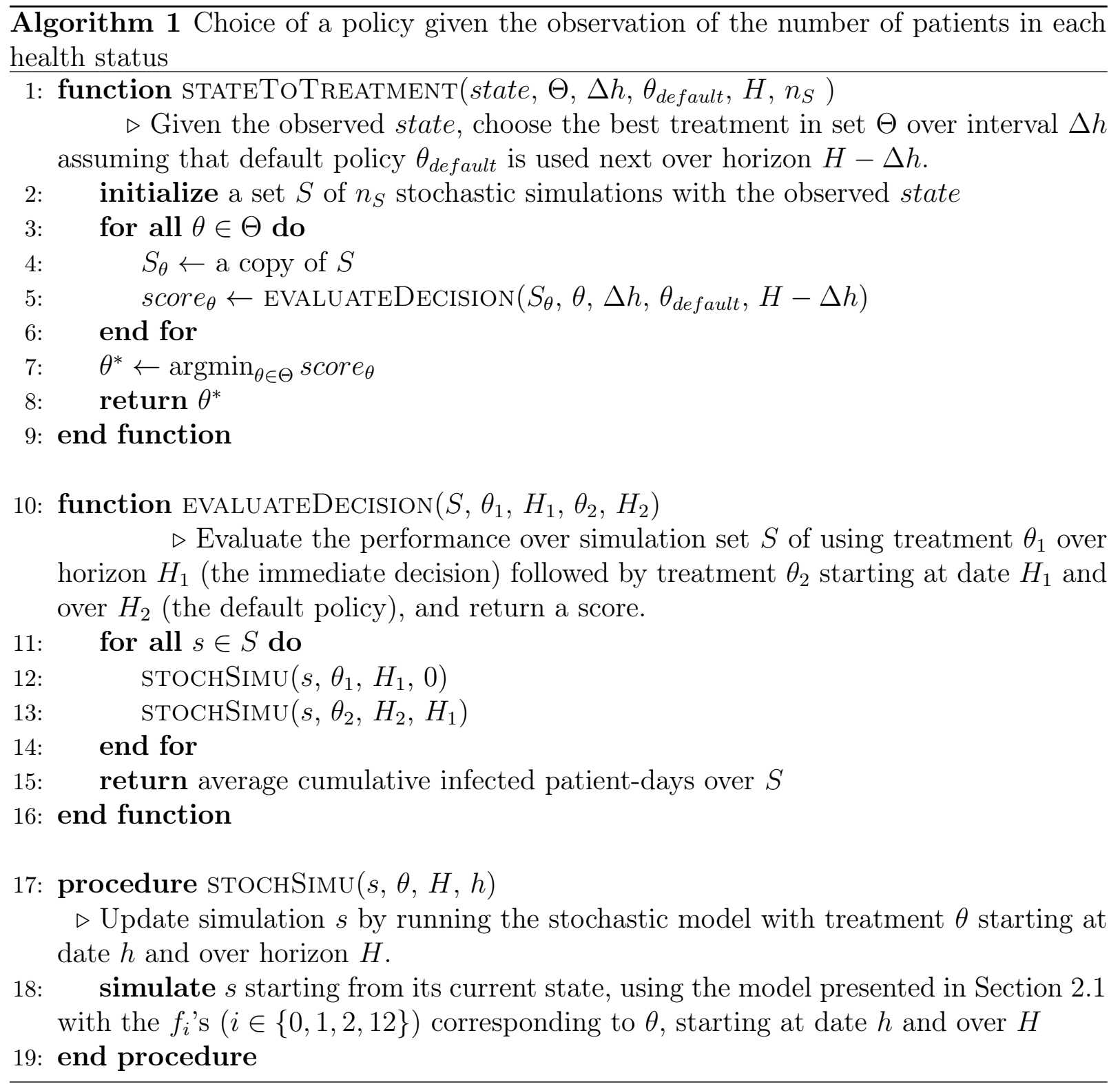




\section{Results}

We ran 400 simulations using the optimization algorithm presented in Section 2.2. Each simulation was initialized with a period of 30 years (10800 days) without treatment. A treatment was then chosen for each period of $\Delta h=15$ days over two years (720 days) by observing the number of patients at the beginning of the period and calling function StateToTreatment. Thus, we obtained 400 different hospital-wide treatment regimens (shown in Figure A.2 in Appendix).

We call OPTIDYN the therapy policy that consists in using our optimization algorithm to choose treatments. We compare the performance of OPTIDYN over two years with that of the following policies: NONE, that consists in administering no treatment; COMBO, that consists in administering treatment 12; CYC-30, that consists in alternating 30 days of treatment 1 with 30 days of treatment 2; MONO-1 that consists in treatment 1 in monotherapy; and MONO-2, that consists in treatment 2 in monotherapy. Finally, we compare OPTIDYN with OPTI, a policy that consists in choosing a treatment regimen for two years with drug switches allowed every 30 days, based on no other information than the population dynamics parameters, as explained in [11]. ${ }^{4}$

The results obtained with the considered policies are shown in Figure 2 and Table 3. The combination therapy COMBO and the cycling therapy CYC-30 show relatively close performances. The former allows a $32.4 \%$ and the latter a $31.2 \%$ reduction in the average cumulative infected patient-days over two years compared to the NONE policy. Both COMBO and CYC-30 perform better than the monotherapies MONO-1 and MONO-2. The "optimal" policy obtained without health status counts, OPTI, allows to decrease the average cumulative infected patient-days by $47.1 \%$ compared to NONE, and by $21.8 \%$ compared to COMBO. By comparison, OPTIDYN yields a reduction of $64.1 \%$ compared to NONE, and of $47.0 \%$ compared to COMBO. Finally, OPTIDYN allows a reduction of $32.2 \%$ in the average cumulative infected patient-days compared to OPTI. The reductions in patient-days obtained with OPTIDYN are significant at the $95 \%$ threshold.

In Figure 3, we compare the evolution in time of the average cumulative infected patientdays under OPTI, COMBO, and OPTIDYN (see Figure A.7 in Appendix for percentiles). OPTI and COMBO tend to yield close results until approximately day 250. The performance of OPTIDYN, however, diverges from that of OPTI and COMBO as early as from day 100. Hence during the first 100 days, the information regarding the state of the epidemic is of little value - this period corresponds to the transient state departing from the initial treatment 0 steady state. However, after day 100, information has a significant value as it

\footnotetext{
${ }^{4}$ See Figures A.3-A.6 in Appendix for the evolution in time of the population in each compartment under different policies.
} 


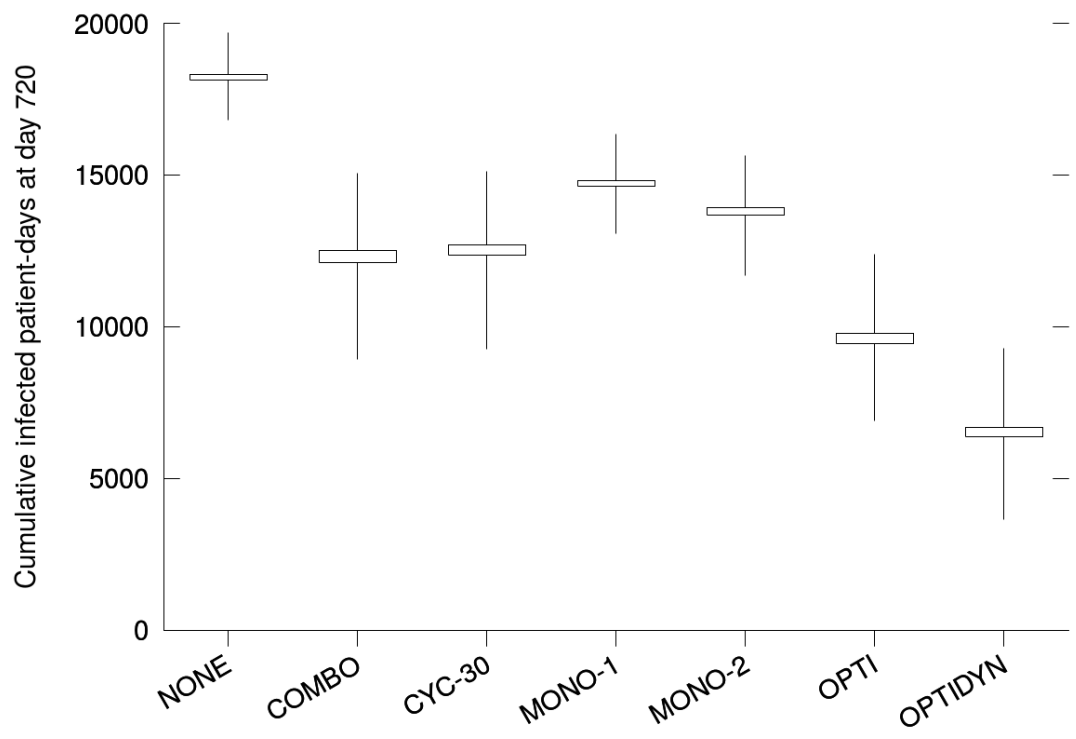

Figure 2: Box plot of the cumulative infected patient-days (400 simulations). Boxes: $95 \%$ confidence intervals. Whiskers: $5^{\text {th }}$ and $95^{\text {th }}$ percentiles of the simulations.

\begin{tabular}{lcc}
\hline Policy & Patient-days & $\mathbf{9 5 \%}$ CI \\
\hline NONE & $18,215.4$ & $18,126.2-18,304.6$ \\
COMBO & $12,314.5$ & $12,122.3-12,506.8$ \\
CYC-30 & $12,525.3$ & $12,355.1-12,695.6$ \\
MONO-1 & $14,716.7$ & $14,618.9-14,814.6$ \\
MONO-2 & $13,813.8$ & $13,689.7-13,937.9$ \\
OPTI & $9,629.61$ & $9,462.96-9,796.25$ \\
OPTIDYN & $6,531.13$ & $6,365.9-6,696.36$ \\
\hline
\end{tabular}

Table 3: Average cumulative infected patient-days (400 simulations) obtained with different policies.

allows a substantial decrease in the number of infected individuals.

We also ran simulations using our algorithm to compute OPTIDYN with $\Delta h=30$ days and $\Delta h=5$ days. The results are shown in Table 4 . Starting from optimization without surveillance and treatment switches allowed every 30 days (OPTI policy, see Table 3), implementing surveillance every 30 days and using this information to decide on treatments allows a $23.4 \%$ decrease in average cumulative infected patient-days over two years. Doubling surveillance frequency to $\Delta h=15$ days yields a further $11.5 \%$ decrease. With $\Delta h=5$ days, the stochastic noise is too important to have better results than with $\Delta h=15$ days and the performance is decreased. Improving this result would require larger computational power. 


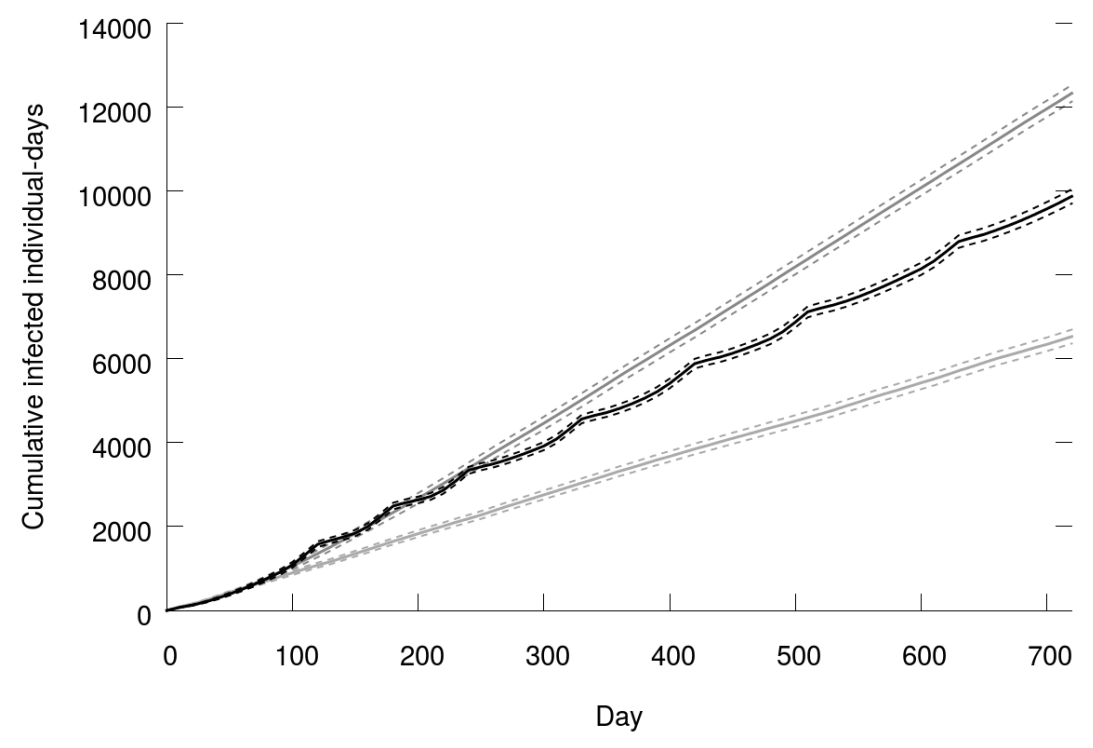

Figure 3: Average cumulative infected patient-days as a function of time (400 simulations). Black: OPTI. Gray (above): COMBO. Gray (below): OPTIDYN. Dashed: 95\% CI.

\begin{tabular}{ccc}
\hline$\Delta h$ & Patient-days & $\mathbf{9 5 \%} \mathbf{C I}$ \\
\hline 30 days & $7,378.65$ & $7,196.13-7,561.17$ \\
15 days & $6,531.13$ & $6,365.9-6,696.36$ \\
5 days & $8,639.12$ & $8,420.17-8,858.06$ \\
\hline
\end{tabular}

Table 4: Average cumulative infected patient-days (400 simulations) obtained with OPTIDYN and different values of $\Delta h$.

In a health care facility, our optimization algorithm can be implemented online by screening the patient population to get the number of patients with each health status every $\Delta h$ days, and then calling function STATETOTREATMENT to decide on a treatment for the next $\Delta h$ days. The computation of a treatment for the next $\Delta h$ days given the current state of the epidemic is tractable on a laptop computer. An alternative way to use the algorithm is to run simulations beforehand (just as we did to produce the results presented here), store the results, and use them to read directly the treatment to be administered given the population with each health status, and the population evolution to be expected given that treatment. Doing so, it might prove necessary to resort to clustering techniques in order to recognize what treatment to use at a given time given the number of individuals with each health status. Deep learning algorithms, for instance, have been used to solve complex recognition tasks. ${ }^{5}$ The implementation of these algorithms is

\footnotetext{
${ }^{5}$ We illustrate the complexity of the output data of our model in Figure A.8 in Appendix.
} 
left to later investigations.

\section{Conclusion}

We propose a solution to the problem of choosing an antimicrobial treatment to be administered to the whole patient population in a health care facility. Our objective is to minimize the cumulative infected patient-days in the long run given that infecting organisms may evolve antimicrobial resistance. The treatment is to be chosen periodically based on the count of patients with each health status at that moment, and on the population dynamics parameters of the health care facility and of the disease. Previous studies have investigated empirical strategies based on screening test results. The great advantage of such strategies is that they make use of information available at no additional cost. However this approach typically only allows to compare a number of specific predefined strategies, such as threshold-based rules in which empirical treatments are switched if resistance frequency reaches some prespecified level. Also, these strategies are adaptive in that they specify a treatment at each time without taking account of the subsequent spread of the disease.

To some extent, it could be said that practical considerations (such as the availability of screening data) were the starting point of these previous studies. Here we looked at the problem the other way around. We strove for near optimal treatments as a first step, using a flexible method that will allow to take any practical or ethical constraint into account in future studies. To do this we used a Monte-Carlo look-ahead method that consists in a choosing a treatment at a given time based on a large number of stochastic simulations heuristically assuming future treatments.

In the scenario considered in this article, our method allowed a $47.0 \%$ reduction in the average cumulative infected patient-days over two years compared to the combination therapy (a strategy widely used in the clinic). Compared to an optimization method that does not use periodic surveillance data, the average cumulative infected patient-days was reduced by $32.2 \%$.

Our algorithm can be used to produce data amenable to quantitative analysis. We believe that this calls for further investigation as it could allow to set up simple and practical decision rules to solve the complex problem of choosing treatments to be administered to all patients in a health care facility.

As a word of caution, we must emphasize that just as any other simulation-based approach, or method is only as good as the underlying epidemiological model. Besides, in reallife settings, model parameters are often unknown or difficult to obtain. Yet our method is compatible with advanced epidemiological models (e.g. agent-based) and Bayesian model 
calibration techniques.

Finally, our method is essentially heuristic. By no mean do we claim that it will outperform much simpler methods (simple feedback controllers, say) in all settings. However, the emergence and spread of antimicrobial resistance being a highly complex phenomenon, we are confident that heuristic Monte-Carlo methods will suit real-life settings. 


\section{References}

[1] B Allegranzi, Roberto Luzzati, A Luzzani, F Girardini, L Antozzi, R Raiteri, Giovanni Di Perri, and E Concia. Impact of antibiotic changes in empirical therapy on antimicrobial resistance in intensive care unit-acquired infections. Journal of Hospital Infection, 52(2):136-140, 2002.

[2] Dan I. Andersson and Diarmaid Hughes. Antibiotic resistance and its cost: is it possible to reverse resistance? Nature Reviews Microbiology, 8(4):260, 2010.

[3] Robert Eric Beardmore, Rafael Peña-Miller, Fabio Gori, and Jonathan Iredell. Antibiotic cycling and antibiotic mixing: which one best mitigates antibiotic resistance? Molecular biology and evolution, 34(4):802-817, 2017.

[4] Carl T Bergstrom, Monique Lo, and Marc Lipsitch. Ecological theory suggests that antimicrobial cycling will not reduce antimicrobial resistance in hospitals. Proceedings of the National Academy of Sciences, 101(36):13285-13290, 2004.

[5] Dimitri P Bertsekas. Rollout algorithms for constrained dynamic programming. Lab. for Information and Decision Systems Report, 2646, 2005.

[6] François Blanquart. Evolutionary epidemiology models to predict the dynamics of antibiotic resistance. Evolutionary applications, 12(3):365-383, 2019.

[7] Sebastian Bonhoeffer, Pia Abel zur Wiesch, and Roger D Kouyos. Rotating antibiotics does not minimize selection for resistance. Math Biosci Eng, 7(4):919-22, 2010.

[8] Alessandro Cassini, Liselotte Diaz Högberg, Diamantis Plachouras, Annalisa Quattrocchi, Ana Hoxha, Gunnar Skov Simonsen, Mélanie Colomb-Cotinat, Mirjam E Kretzschmar, Brecht Devleesschauwer, Michele Cecchini, et al. Attributable deaths and disability-adjusted life-years caused by infections with antibiotic-resistant bacteria in the eu and the european economic area in 2015: a population-level modelling analysis. The Lancet Infectious Diseases, 19(1):56-66, 2019.

[9] Justin C Goodson, Barrett W Thomas, and Jeffrey W Ohlmann. A rollout algorithm framework for heuristic solutions to finite-horizon stochastic dynamic programs. European Journal of Operational Research, 258(1):216-229, 2017.

[10] Nicolas Houy and Julien Flaig. Informed and uninformed empirical therapy policies. Mathematical Medicine and Biology: A Journal of the IMA, 122019. 
[11] Nicolas Houy and Julien Flaig. Optimal dynamic empirical therapy in a health care facility: a monte-carlo look-ahead method. Computer Methods and Programs in Biomedicine, page 105767, 2020.

[12] Nicolas Houy and François Le Grand. Optimizing immune cell therapies with artificial intelligence. Journal of Theoretical Biology, 461:34-40, jan 2019.

[13] Nicolas Houy and François Le Grand. Optimal dynamic regimens with artificial intelligence: The case of temozolomide. PLOS ONE, 13(6):1-15, 062018.

[14] Annette Jepson. Microbiology and infection control. In Carlos M H Gómez, editor, Clinical Intensive Care Medicine, chapter 10. Imperial College Press, 2014.

[15] Roger D. Kouyos, Pia Abel zur Wiesch, and Sebastian Bonhoeffer. Informed switching strongly decreases the prevalence of antibiotic resistance in hospital wards. PLoS computational biology, 7(3):e1001094, 2011.

[16] Bruce R Levin and Marc JM Bonten. Cycling antibiotics may not be good for your health. Proceedings of the National Academy of Sciences, 101(36):13101-13102, 2004.

[17] Marc Lipsitch and Matthew H. Samore. Antimicrobial use and antimicrobial resistance: a population perspective. Emerging infectious diseases, 8(4):347-354, 2002.

[18] Anita H. Melnyk, Alex Wong, and Rees Kassen. The fitness costs of antibiotic resistance mutations. Evolutionary applications, 8(3):273-283, 2015.

[19] Michael S. Niederman. Is "crop rotation" of antibiotics the solution to a "resistant" problem in the icu? American Journal of Respiratory and Critical Care Medicine, 156(4):1029-1031, 1997.

[20] Anna Maria Niewiadomska, Bamini Jayabalasingham, Jessica C Seidman, Lander Willem, Bryan Grenfell, David Spiro, and Cecile Viboud. Population-level mathematical modeling of antimicrobial resistance: a systematic review. BMC medicine, 17(1):1-20, 2019.

[21] R Peña-Miller and Robert Beardmore. Rotating antibiotics selects optimally against antibiotic resistance, in theory. Mathematical Biosciences \& Engineering, 7(3):pp. 527-552, 2010.

[22] Rafael Peña-Miller and Robert Beardmore. Antibiotic cycling versus mixing: the difficulty of using mathematical models to definitively quantify their relative merits. Mathematical Biosciences \& Engineering, 7(4):923-933, 2010. 
[23] D. E. Ramsay, J. Invik, S. L. Checkley, S. P. Gow, N. D. Osgood, and C. L. Waldner. Application of dynamic modelling techniques to the problem of antibacterial use and resistance: a scoping review. Epidemiology \& Infection, 146(16):2014-2027, 2018.

[24] Timothy C. Reluga. Simple models of antibiotic cycling. Mathematical medicine and biology: a journal of the IMA, 22(2):187-208, 2005.

[25] Eden L Romm and Igor F Tsigelny. Artificial intelligence in drug treatment. Annual review of pharmacology and toxicology, 60, 2020.

[26] Burcu Tepekule, Hildegard Uecker, Isabel Derungs, Antoine Frenoy, and Sebastian Bonhoeffer. Modeling antibiotic treatment in hospitals: A systematic approach shows benefits of combination therapy over cycling, mixing, and mono-drug therapies. PLoS computational biology, 13(9):e1005745, 2017.

[27] Pia Abel zur Wiesch, Roger Kouyos, Sören Abel, Wolfgang Viechtbauer, and Sebastian Bonhoeffer. Cycling empirical antibiotic therapy in hospitals: meta-analysis and models. PLoS pathogens, 10(6):e1004225, 2014. 


\section{A Additional figures}

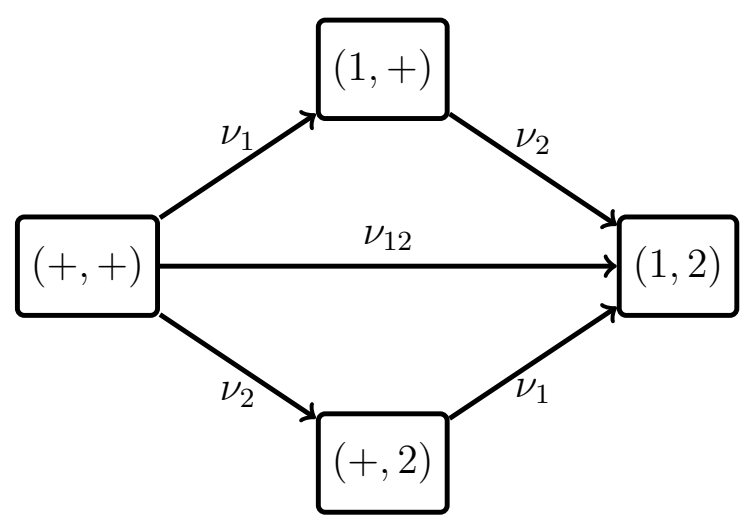

Figure A.1: The four genotypes included in our model with mutation rates. The arrows represent transitions between genotypes. The two considered genes are separated by a comma. '+' represents a wild-type allele. ' 1 ' and '2' represent alleles giving resistance to drugs 1 and 2 respectively. $(+,+)$ : wild-type susceptible. $(1,+)$ : 1 -resistance only. $(+, 2)$ : 2-resistance only. $(1,2)$ : resistance to both drugs. 


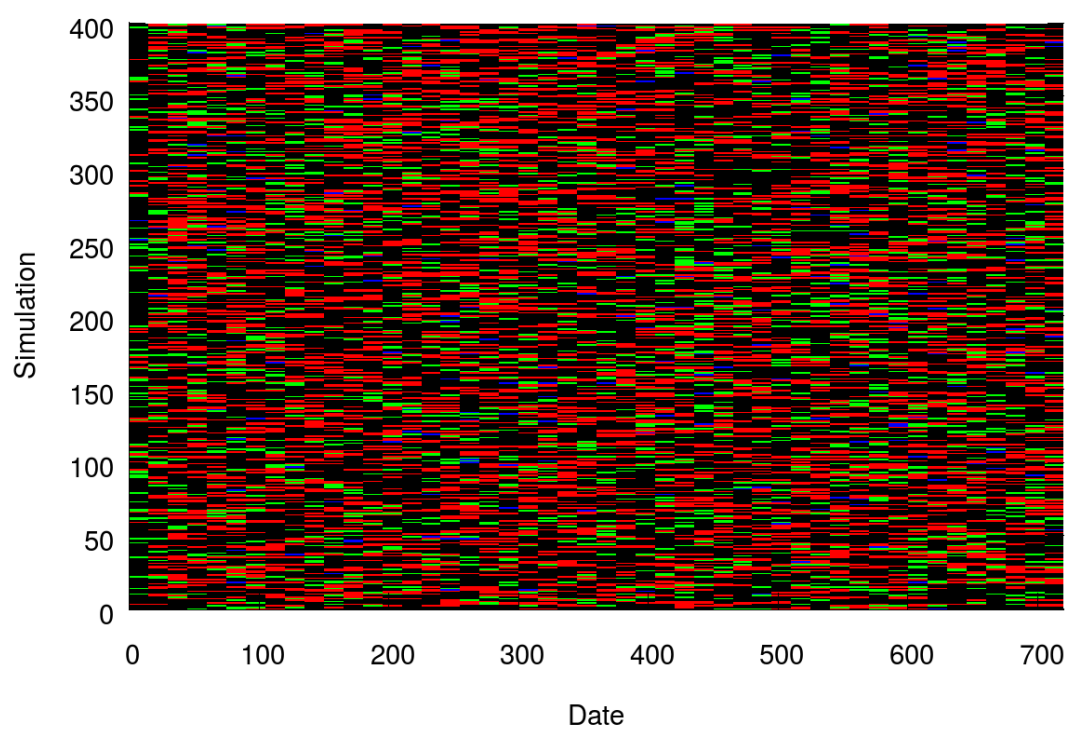

Figure A.2: The 400 treatment regimens obtained with our optimization algorithm. Red: treatment 0. Green: treatment 1. Blue: treatment 2. Black: treatment 12. 


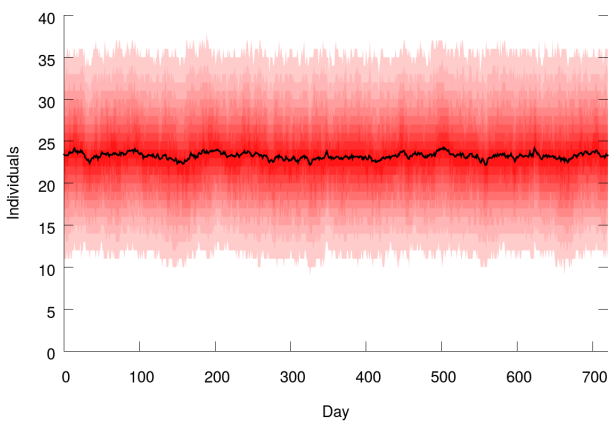

(a) $S$

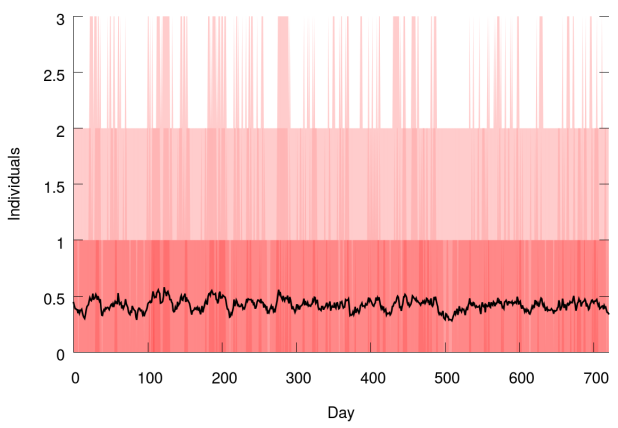

(c) $R_{2}$

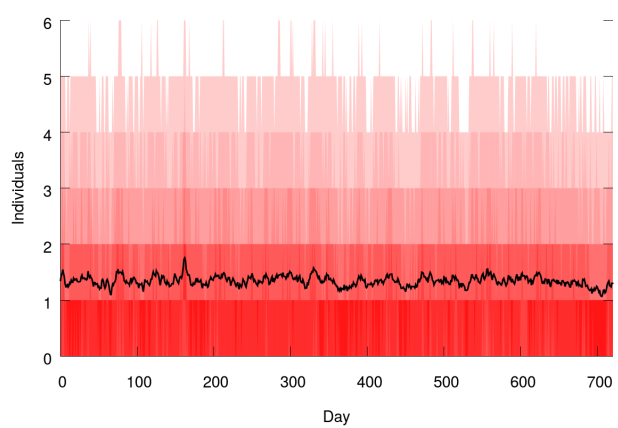

(b) $R_{1}$

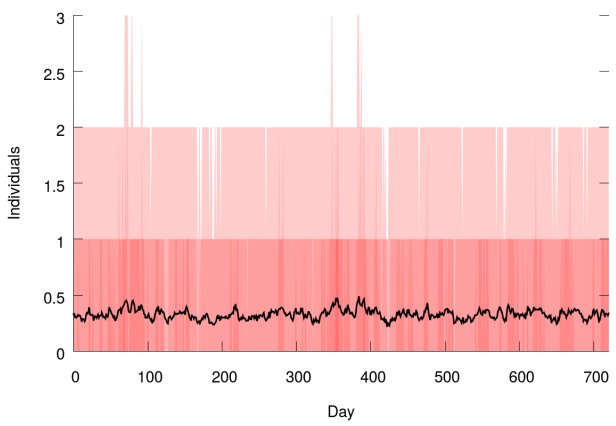

(d) $R_{12}$

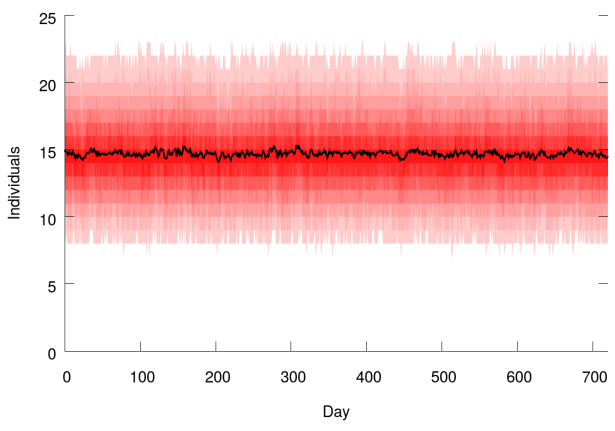

(e) $X$

Figure A.3: Number of patients in each compartment as a function of time when no treatment is used (NONE policy). Black: average over 400 simulations. Shades of red: $i^{\text {th }}$ and $(100-i)^{\text {th }}$ percentiles for $i$ from 5 to 50 by increments of 5 . 


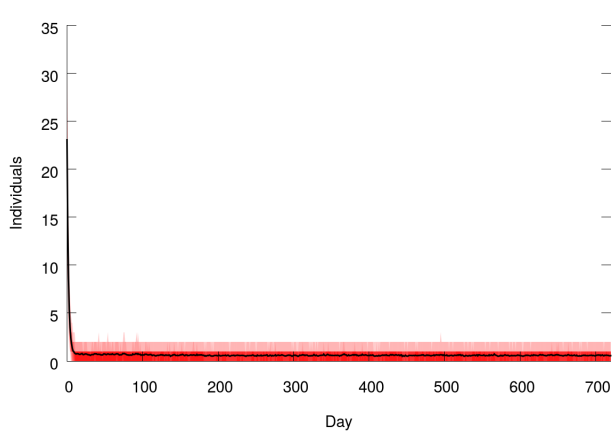

(a) $S$

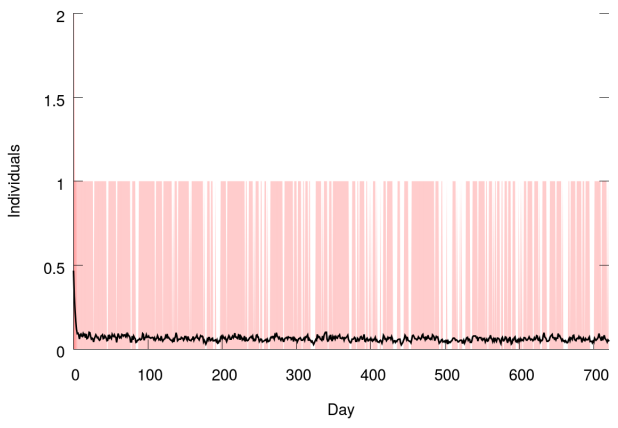

(c) $R_{2}$

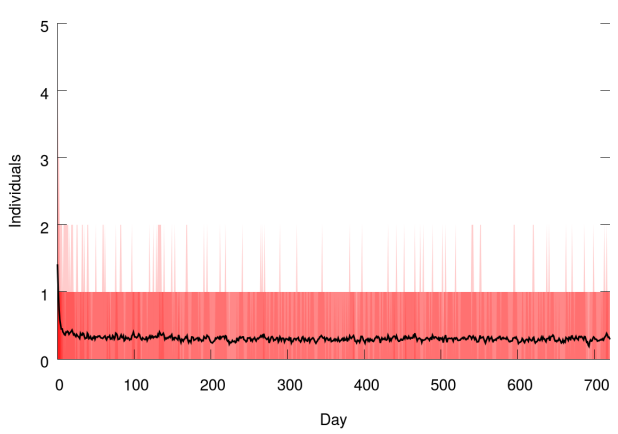

(b) $R_{1}$

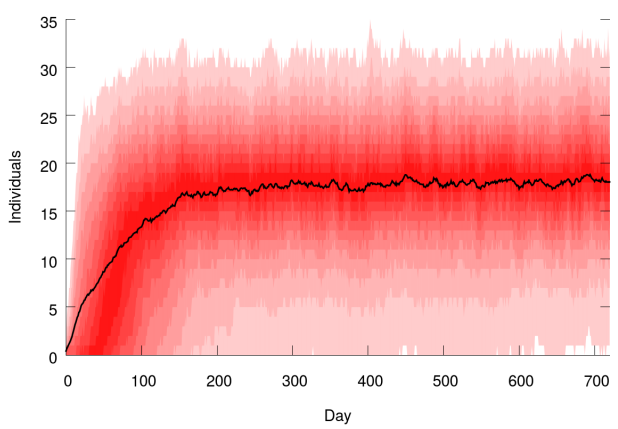

(d) $R_{12}$

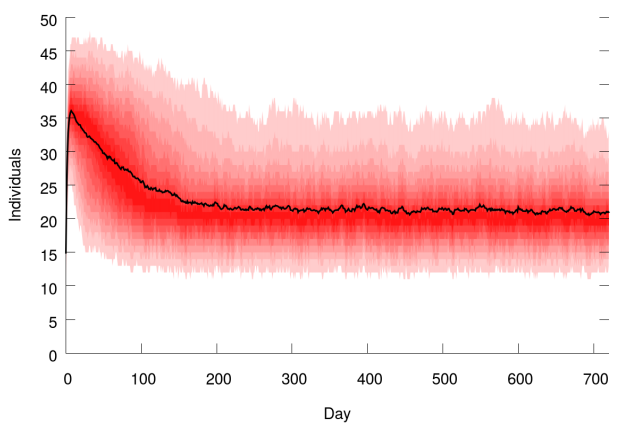

(e) $X$

Figure A.4: Number of patients in each compartment as a function of time when the COMBO policy is used. Black: average over 400 simulations. Shades of red: $i^{\text {th }}$ and $(100-i)^{\text {th }}$ percentiles for $i$ from 5 to 50 by increments of 5 . 


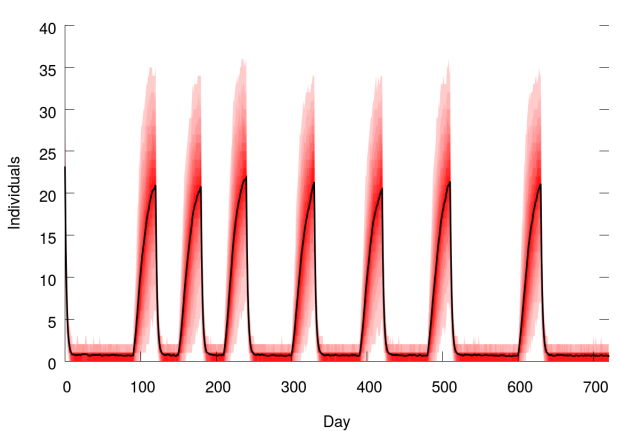

(a) $S$

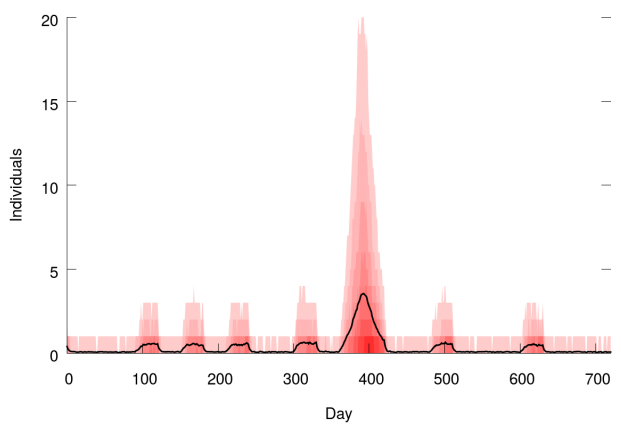

(c) $R_{2}$

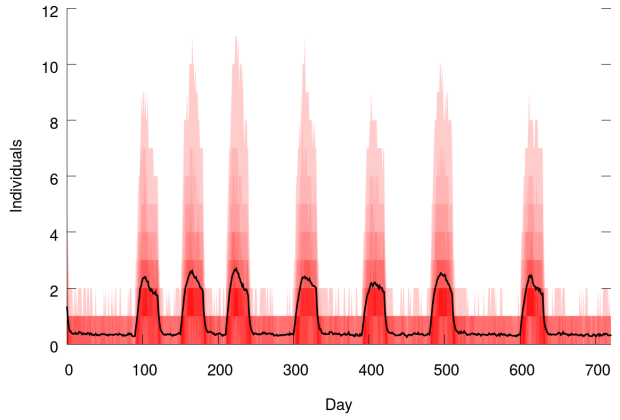

(b) $R_{1}$

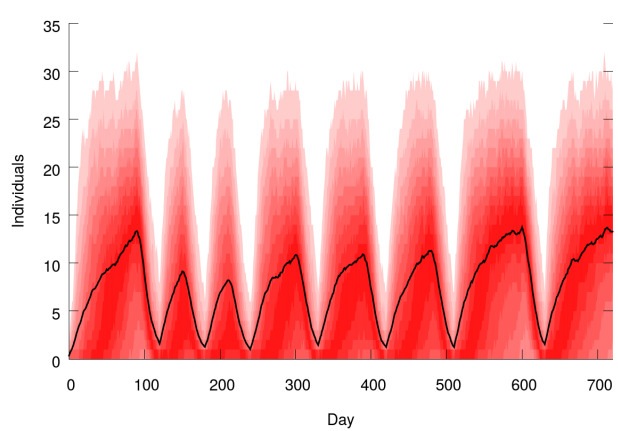

(d) $R_{12}$

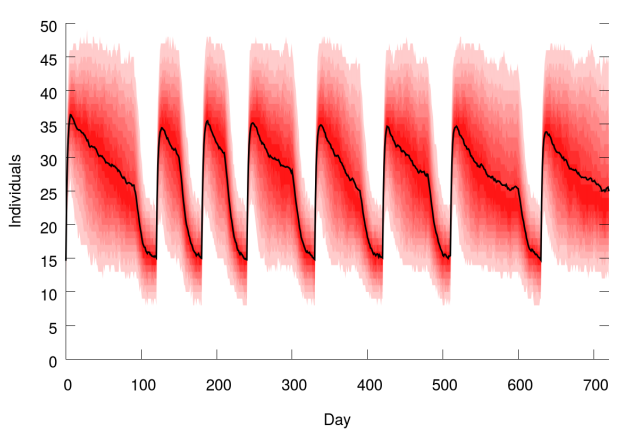

(e) $X$

Figure A.5: Number of patients in each compartment as a function of time when the OPTI policy is used. Black: average over 400 simulations. Shades of red: $i^{\text {th }}$ and $(100-i)^{\text {th }}$ percentiles for $i$ from 5 to 50 by increments of 5 . 


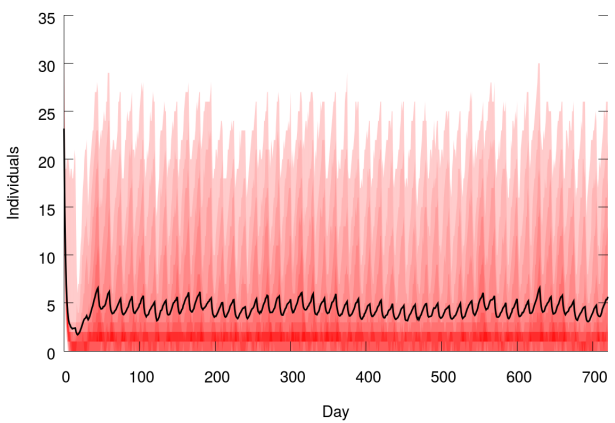

(a) $S$

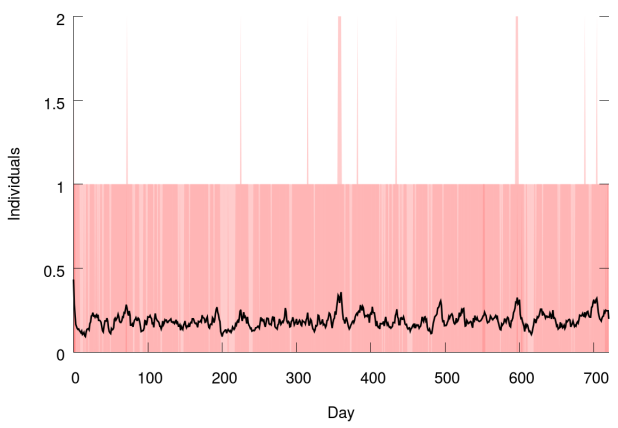

(c) $R_{2}$

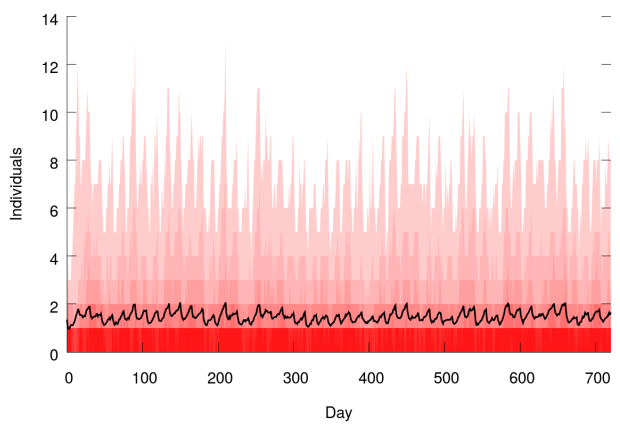

(b) $R_{1}$

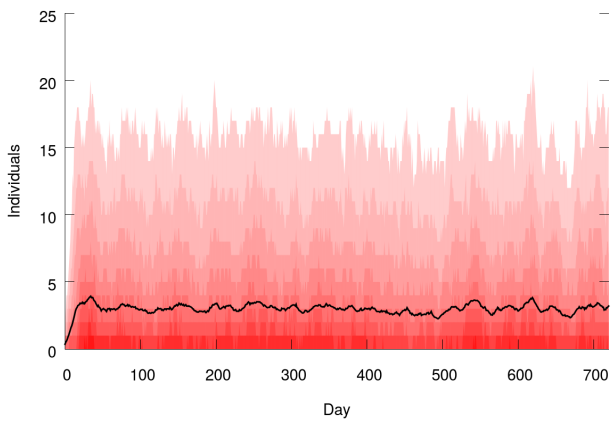

(d) $R_{12}$

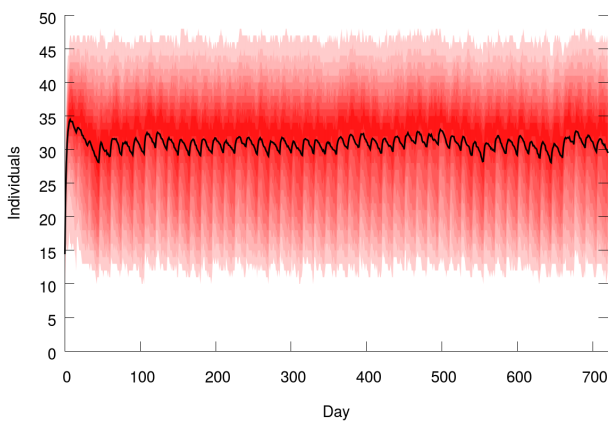

(e) $X$

Figure A.6: Number of patients in each compartment as a function of time when the OPTIDYN policy is used. Black: average over 400 simulations. Shades of red: $i^{\text {th }}$ and $(100-i)^{\text {th }}$ percentiles for $i$ from 5 to 50 by increments of 5 . 


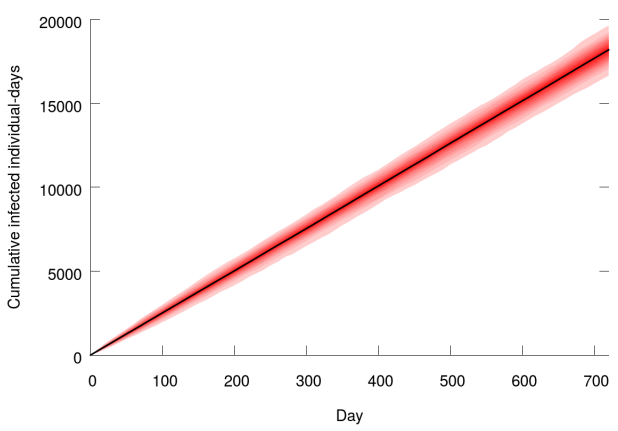

(a) NONE

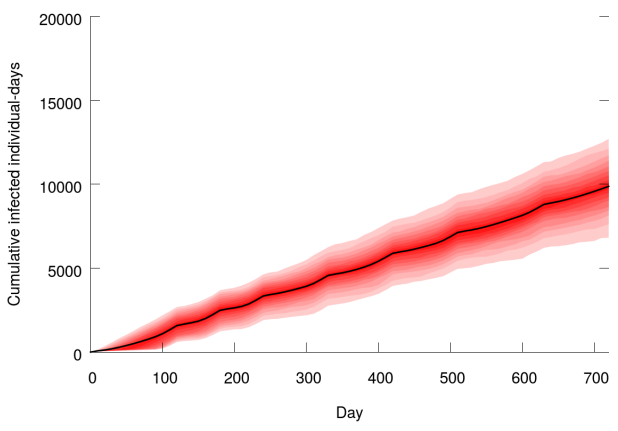

(c) OPTI

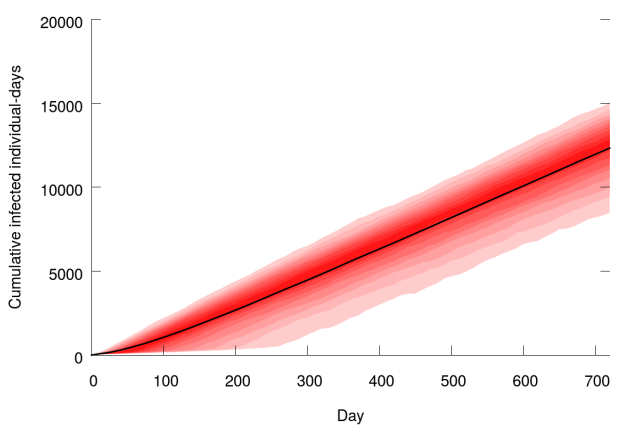

(b) COMBO

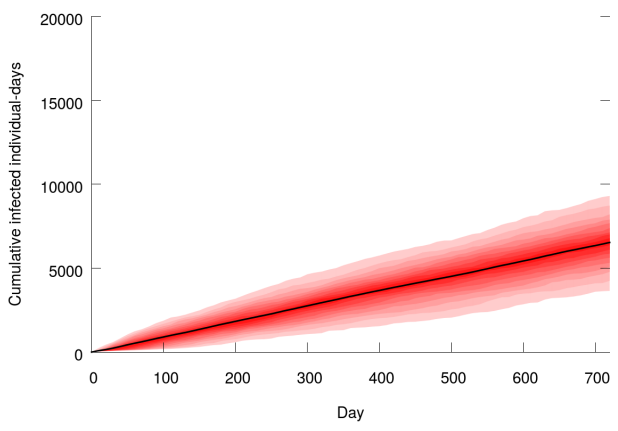

(d) OPTIDYN

Figure A.7: Cumulative infected patient-days as a function time when the NONE, COMBO, OPTI and OPTIDYN policies are used. Black: average over 400 simulations. Shades of red: $i^{\text {th }}$ and $(100-i)^{\text {th }}$ percentiles for $i$ from 5 to 50 by increments of 5 . 


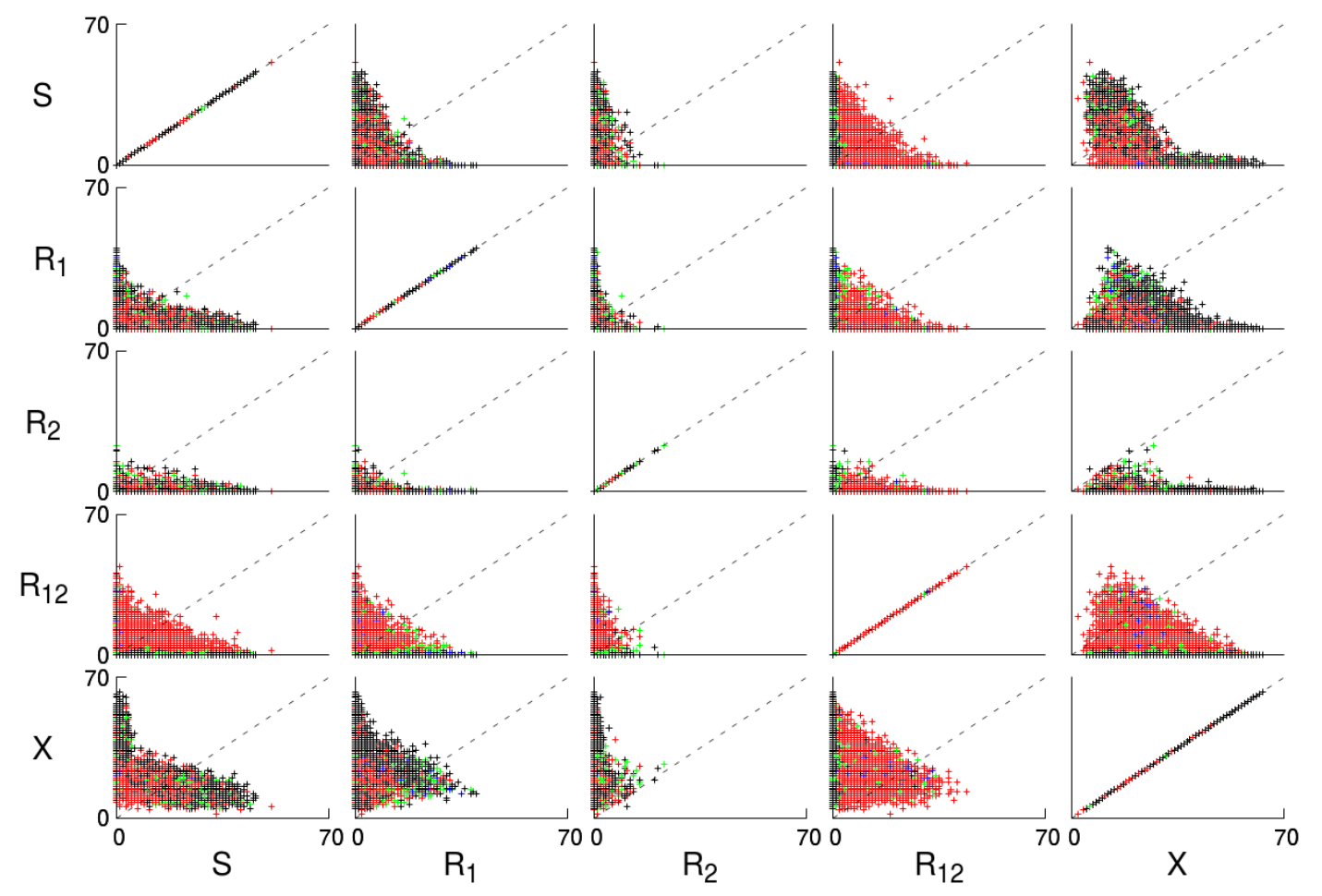

Figure A.8: Chosen treatment as a function of the observed population in pairs of compartments. Crosses: function stateToTreatment calls. Red: treatment 0. Green: treatment 1. Blue: treatment 2. Black: treatment 12. Dashed: $\mathrm{y}=\mathrm{x}$ lines. 